San Jose State University

SJSU ScholarWorks

Master's Theses

Master's Theses and Graduate Research

1992

\title{
Effects of turbulence and activation method on touchscreen performance in aviation environments
}

Kristin G. Bauersfeld

San Jose State University

Follow this and additional works at: https://scholarworks.sjsu.edu/etd_theses

\section{Recommended Citation}

Bauersfeld, Kristin G., "Effects of turbulence and activation method on touchscreen performance in aviation environments" (1992). Master's Theses. 374.

DOI: https://doi.org/10.31979/etd.sgh6-aqgx

https://scholarworks.sjsu.edu/etd_theses/374

This Thesis is brought to you for free and open access by the Master's Theses and Graduate Research at SJSU ScholarWorks. It has been accepted for inclusion in Master's Theses by an authorized administrator of SJSU ScholarWorks. For more information, please contact scholarworks@sjsu.edu. 


\section{INFORMATION TO USERS}

This manuscript has been reproduced from the microfilm master. UMI films the text directly from the original or copy submitted. Thus, some thesis and dissertation copies are in typewriter face, while others may be from any type of computer printer.

The quality of this reproduction is dependent upon the quality of the copy submitted. Broken or indistinct print, colored or poor quality illustrations and photographs, print bleedthrough, substandard margins, and improper alignment can adversely affect reproduction.

In the unlikely event that the author did not send UMI a complete manuscript and there are missing pages, these will be noted. Also, if unauthorized copyright material had to be removed, a note will indicate the deletion.

Oversize materials (e.g., maps, drawings, charts) are reproduced by sectioning the original, beginning at the upper left-hand corner and continuing from left to right in equal sections with small overlaps. Each original is also photographed in one exposure and is included in reduced form at the back of the book.

Photographs included in the original manuscript have been reproduced xerographically in this copy. Higher quality $6 "$ " 9 9" black and white photographic prints are available for any photographs or illustrations appearing in this copy for an additional charge. Contact UMI directly to order.

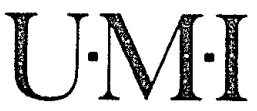

University Microfılms Internatıonal

A Bell \& Howell Information Company 300 North Zeeb Road. Ann Arbor, MI 48106-1346 USA

$313 / 761-4700 \quad 800 / 521-0600$ 

Order Number 1350068

Effects of turbulence and activation method on touchscreen performance in aviation environments

Bauersfeld, Kristin Grace, M.A.

San Jose State University, 1992

Ann Arbor, MI 48106 



\title{
EFFECTS OF TURBULENCE AND ACTIVATION METHOD ON TOUCHSCREEN PERFORMANCE IN AVIATION ENVIRONMENTS
}

\author{
A Thesis \\ Presented to \\ The Faculty of the Department of Psychology \\ San Jose State University \\ In Partial Fulfillment \\ of the Requirements for the Degree \\ Master of Arts
}

by

Kristin G. Bauersfeld

August, 1992 
APPROVED FOR THE DEPARTMENT OF PSYCHOLOGY

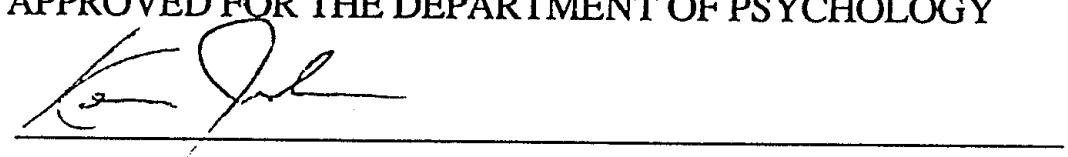

Kevin Jordan PhD., Professor of Psychology, SJSU

Asap Decani

Asa Degani M.S., Research Associate,

San Jose State University Foundation

Robert Cooper

Robert Cooper PhD., Professor of Psychology, SJSU

APPROVED FOR THE UNIVERSITY
th. Sou Leurandowele 


\section{ACKNOWLEDGEMENTS}

I would like to give thanks to Eric Johnston for without his help I would have never gotten through this with my sanity intact. I would also like to thank Asaf Degani, Kevin Jordan, and Robert Cooper for their mentorship and numerous hours of draft reading. Additional thanks to Don Bryant, Barry Sullivan and everyone at the MVSRF who participated in making this a successful simulation. Thanks to Everett Palmer and the Flight Human Factors Branch at NASA Ames Research Center for supporting this work (RTOP \#505-64-13). Thanks to Dennis Beringer for his comments and suggestions. And finally, thanks to all my parents for all their support and encouragement. 


\section{TABLE OF CONTENTS}

SECTION

PAGE

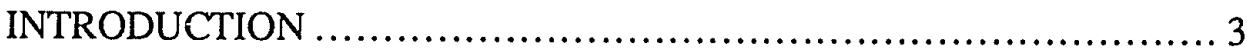

Touchscreens .................................................... 4

Activation Methods..................................................... 4

Turbulence ........................................................... 9

Task Performance and Turbulence ................................13

Objective................................................................

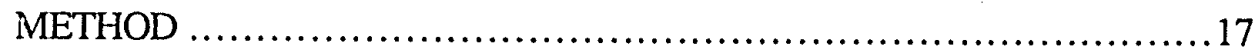

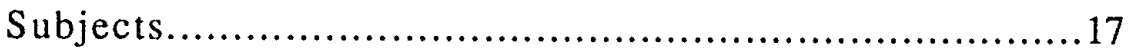

Apparatus............................................................

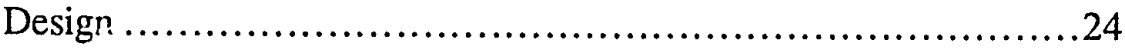

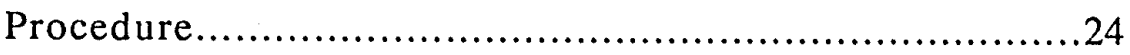

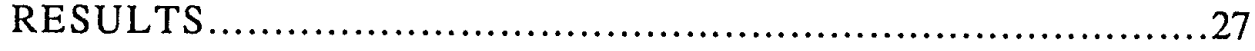

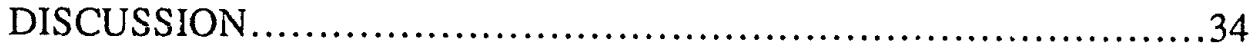

Activation Method................................................. 34

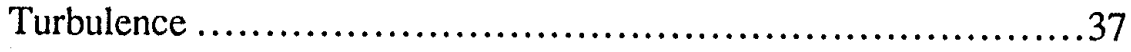

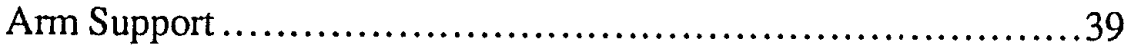

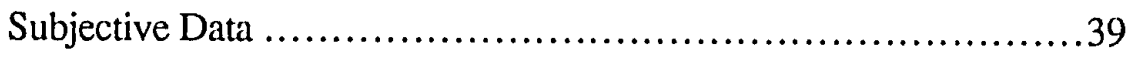

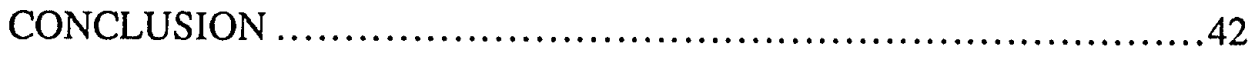

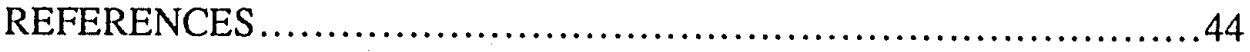

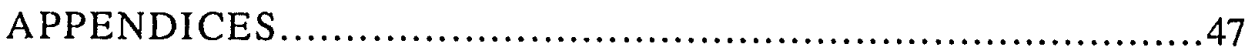

Appendix A. Signed Approval Forms ..............................47 


\section{LIST OF TABLES}

TABLE PAGE

1. Descriptive Statistics. 30

2. Correlations Among Study Variables. 35

3. Variables Which Entered Into The Regression Analyses. 36 


\section{LIST OF FIGURES}

FIGURE

PAGE

1. $\mathrm{X}, \mathrm{Y}, \mathrm{Z}$ co-ordinate system (adopted from Griffin, 1990).

2. Taxonomic model of human operator processes

contributing to performance with vibration. Adapted

from Lewis and Griffin $(1976,1978)$.

3. View of the Advanced Concepts Flight Simulator.

4. Summary of the anthropometric relations between

the pilots and the displays in the cockpit of the Advanced Concepts Flight Simulator.

5. Touchscreen measurements along with touchcell grid and $x, y$ dimensions.

6. An example of a checklist button, grey areas define active areas.

7. A figure showing the checklist or malfunction generating the checklist and the resulting approximate number of button touches (\#).

8. Main effect of activation method on duration

9. Interaction between activation method and turbulence for duration.

10. Main effect of turbulence on path length.

11. How did the severity of turbulence in the simulator compare to turbulence you experience while flying 
on the line?

40

12. How difficult was it to use the touchscreens during

turbulence in the simulator? 
Touchscreens and Turbulence

\author{
Effects of Turbulence and Activation Method on \\ Touchscreen Performance in Aviation Environments \\ Kristin G. Bauersfeld \\ San Jose State University
}

\title{
Running head: TOUCHSCREENS AND TURBULENCE
}

\author{
Requests for reprints should be sent to Kristin Bauersfeld, Department of Psychology, San \\ Jose State University, San Jose, California 95192.
}


Touchscreens and Turbulence

\begin{abstract}
A study was conducted to determine the effects of varying levels of turbulence on airline pilot performance while using touchscreens. The goal of the present study was to determine what factors influence finger movement on the screen, and which activation method (first-contact or lift-off) was the most effective method in terms of speed and accuracy. Two levels of turbulence (medium and high) and a control condition (no turbulence) was used. A main effect of activation method on finger curation on the screen (duration) was found $F(1,14)=4.26, p<.058 ; \hat{W^{2}}=.16$. An interaction between activation method and turbulence was found for duration $F(2,28)=3.27, p<.053 ; \hat{W}^{2}=$ .12. A main effect of turbulence on path length (the distance the finger traveled on the screen) was found $F(2,28)=3.56, p<.05$. Results indicate that a trade-off exists between the two types of activation methods during turbulence. Further results reveal that although finger movement increased as turbulence level increased, the movement did not impair the pilots' ability to perform tasks.
\end{abstract}


Touchscreens and Turbulence

Effects of Turbulence and Activation Method on Touchscreen Performance in Aviation Environments

While touchscreen technologies have been widely used for some time, the applications have been limited to highly error-tolerant environments (i.e., lottery machines, automatic bank tellers, shopping mall directories). More current applications have found touchscreens being used in highly dynamic task environments such as helicopter and airplane cockpits. Thicre has been only limited study of the effects of touchscreen use in these more demanding, high-risk environments. Of particular concern are issues of vibration-induced errors caused by turbulence and mechanical components in the aircraft as well as errors resulting from the choice of activation method. The purpose of the present study is to address the effects of vibration and activation method on touchscreens used as the primary interface in commercial aviation systems. This will be accomplished through the use of actual pilots, doing actual tasks while flying a realistic scenario. In studying these issues in a realistic environment, we hope to gain an understanding of touchscreen effectiveness in the type of high-risk environments where present touchscreen technology exists and where future touchscreen implementation is likely.

One example of touchscreen technology use in a high risk environment is the McDonnell Douglas F/A-18E/F fighter aircraft. The F/A-18E/F uses two $8 \times 8$ inch touchscreen overlays to display tactical situation, communication, and navigation information (Morrocco, 1991). In the public domain, there are no empirical data available to support the implementation of touchscreens in such a dynamic and high-risk environment. It is possible that no such data exist anywhere. This implementation of touchscreen technologies without human factors evaluation is just one example of the need 
Touchscreens and Turbulence

for a more rigorous examination of touchscreen technologies and their appropriate applications in aviation.

\section{Touchscreens}

Touchscreens provide not only a more direct means of data selection and entry, but they are also faster, easier to use, and more flexible. When empirically compared to other input devices such as mice, lightpens, keyboards and joysticks, touchscreens have been shown to provide for faster selection of stationary targets (Karat, McDonald, \& Anderson, 1986). However, touchscreens are not without problems. Some potential disadvantages of touchscreens are activation method, arm fatigue, limited tactile feedback, and limited visual feedback from low resolution, glare, and smudges (Sears, Plaisant, \& Shneiderman, 1991). Another disadvantage cited by Sears et al. (1991) is parallax, which results in a perceived change in an object's location as a result of viewing position, thereby causing a subject to fall short or overshoot a target. Most of these disadvantages have been overcome with advancements in touchscreen technology. These include advancements in hardware, interface design, and the implementation of alternative activation methods for target selection (Sears \& Shneiderman,1991).

\section{Activation Methods}

Activation method (also referred to in the literature as selection strategy) refers to the manner by which an operator activates a desired target on a touch panel. An example would be the selection of a computer-generated button by pressing down and then lifting the finger off the screen to activate the target. There are five activation methods which are most commonly used as a means of target activation with touchscreen technology: land-on, first-contact, lift-off, land-on -- lift-off, and sequential touch (Sears et ai., 1991). Although many of these activation methods may appear similar in concept and purpose, 
they are quite different in application. Recent research has provided data on the types and effectiveness of activation methods (Sears et al., 1991; Whitfield, Ball \& Bird, 1983), but few of these methods have been evaluated in a high-precision, dynamic task environment. In a high-precision environment, such as an airplane cockpit, it is important to use an activation method which gives the user flexibility and error tolerance in addition to precision and speed in task execution.

"Land-On -- Lift-Off" and "Sequential Touch". With the "Land-on -- Lift-off" method of activation, a user's finger must land on and lift off of the same target in order to complete a selection. A sequence of touches must be performed to complete a selection using "sequential touch" method of activation. A sequence might include first touching a desired target and then touching a separate "confirmation button" to activate the target. Land-on -- lift-off and sequential touch are two methods for which additional confirmation is needed before activation of the target takes place. The additional "confirmation" can be in the form of either exact landing and lifting off the same target (Land-on -- lift-off) or from a sequence of touches (sequential touch). These methods of target selection are particularly effective in critical situations where the consequences of an action might be irreversible. Although these strategies might merit use in a cockpit in specific instances (such as an engine shutdown), these methods require more time and attention than some of the other strategies, making them impractical for many uses.

"Land-On". The "land-on" method, perhaps the most traditional of all the activation methods, is the method in which the first target the user's finger contacts is the selected target. This means that the user is unable to drag his/her finger to a desired target. If the desired target is missed the first time, the user must remove his/her finger from the screen and try again. The user is unable to correct for erroneous touches, making activation of 
Touchscreens and Turbulence

targets in dynamic environments (such as turbulence) quite precarious. This activation method does not allow the speed or error tolerance riecessary when performing tasks in a demanding environment. For example, attempting to select a target on the screen during turbulence could cause jolts to the finger thereby making erroneous target selections more likely. Another activation method, "first-contact", is similar in concept to land-on, but may provide a more suitable application to an airplane cockpit.

"First-Contact". The first-contact method selects the first target the user's fingers come in contact with. If the user touches a target on the first try, that target is selected. If a target is missed on initial contact, the user is able to drag his/her finger to a target. A modified version of this method uses a crosshair which emulates a finger's movement on the screen. Through the use of continuous sensing, the computer is able to determine the user's position on the screen. Therefore, if all targets are missed when the user first touches the screen, he/she can then drag a finger to a specific target and it will be selected. The first target to come in contact with the user's finger, while the finger is being dragged, becomes the selected item. The user removes his or her finger from the screen once a selection has been made in order to prepare for the next selection. This method is one of the more direct and rapid methods, but allows for corrections only if the initial touch was unsuccessful in selecting any of the targets (Beringer \& Lee, 1988). Because the firstcontact method of target activation uses continuous sensing, it makes the method more suitable than land-on for a dynamic task environment because of its error-reccvery ability and expedient activation of the target.

"Lift-Off". With the "lift-off" method of activation, target selection is not complete until the user's finger is removed from the screen. If a target is missed on initial contact, the user is able to drag his/her finger to a target. Like first-contact, the lift-off method 
Touchscreens and Turbulence

enlists the help of continuous sensing in order to determine the user's position on the screen. This method allows a user to drag a finger to a desired location should the target be missed on the first try. Unlike first-contact, the selection of a target does not take place until the finger has been removed from the screen. The last point touched before the removal of the finger from the screen becomes the selected item and the target is activated upon lift-off of the user's finger from the screen. This activation method allows for easy error recovery because of its use of continuous sensing and because a target is not selected until after the finger is removed thus allowing the user to move around the screen untii a desired target is selected.

Of the previously mentioned activation methods, only "lift-off" and "first-contact" appear suitable for use in an aviation environment and therefore, are of primary interest to the present experiment. Both of these activation methods have been shown to be somewhat flexible, error-tolerant, precise, and expedient. However, subtle differences in execution of these two methods requires further analysis.

Comparison of Activation Methods. The feature that sets the "first-contact" and "liftoff" activation methods apart from the other methods is their ability to continually sense a finger's position on the screen. Continuous sensing serves an aviation environment by allowing for error recovery. However, the lift-off method is a little more error-tolerant in that the finger can pass over targets and select only that target from which the finger lifts off. Additionally, lift-off can provide feedback to the user in the form of a visually "3D" button which appears to "pop" in when the button is touched and "pop" out when lifted off, much like a mechanical button would. In contrast, the first-contact method, although having less feedback and error-tolerance, provides a more expedient response to the user's action. Potter, Weldon, and Shneiderman (1988) found that the first-contact method of 
Touchscreens and Turbulence

activation had a significantly faster target activation time than the lift-off method during laboratory experiments. However, the lift-off method had significantly fewer errors than first-contact. Of those errors, lift-off had fewer wrong target errors than first-contact.

Both the first-contact and lift-off methods have been evaluated for their performance on varying target layouts including targets which were both small and large, densely and sparsely placed, and implemented with various types of touchscreens. The lift-off method has been shown to be more effective than first-cuntact wich small targets in censely-packed areas (Sears et al., 1991). In comparison, the first-contact method allows for the effective selection of small targets as long as the targets are placed sparsely on the screen. A potential drawback to the lift-off strategy may occur due to the rolling of the finger pad during withdrawal, which can result in the last point of contact being changed unknowingly by the user. This phenomenon has been termed "fall-out error" (Whitfield et al., 1983). In the present study, a modified lift-off strategy will extend the active area $1 / 8$ inch around the target just before lift-off in an attempt to control for this error.

In general, past research seems to show that the effectiveness of either method depends on the placement and the size of the targets, type of activation method (Potter, Berman \& Shneiderman, 1989; Potter et al., 1988), and type of touchscreen technology used. Due to the realistic environment of the present study, the sizes and densities of the targets are not constant; therefore, it is difficult to assume that one method would be superior to another.

Past research has not compared the efficacy of various activation methods in low errortolerant environments or in the presence of random motion. Most of the research conducted has taken place in laboratory settings where the consequences of errors are relatively minimal; therefore it is difficult to generalize the results of these activation method studies to the high-risk environment of the aviation industry. Studying the lift-off and first-contact 
activation methods through the use of realistic simulations will give a better indication of their actual thresholds of error tolerance. Accurate knowledge of the error tolerance of these methods will then allow for safer implementation into commercial cockpits.

Turbulence

An additional important goal of this study is to determine the effects of turbulence on touchscreen performance in an aviation environment. Lack of research in this area makes it difficult to formulate empirically-driven hypotheses regarding touchscreen performance in random motion environments. However, research on the effects of vibration on manual control tasks and the nature of the task being performed can give an idea of how operators might interact with touchscreens in random motion environments.

It is known that the existence of vibration or turbulence in an environment can interfere with an operator's ability to perform a task (Parks, 1962). However, turbulence effects are generally specific to the nature of the task, the variable nature of environmental vibration, and the variability of individuals, making it difficult to extrapolate data from the laboratory to a real world situation (McLeod \& Griffin, 1986). In a target selection task, Hampton and Dominessey (1989) compared a touchscreen to bezel keys ("keyboard" type keys) during helicopter flight maneuvers. Generalization of their results to settings other than specific rotocraft environments is difficult because they use semantic labels describing helicopter maneuvers to charactcrize vitiational effects instead of numerical acceleration magnitude. Because the present study quantifies the acceleration experienced by the pilots, the present study should result in conclusions that will generalize more readily to actual aviation environments.

Characterizing Turbulence. For the purpose of the present study, the terms vibration and turbulence are interchangeable. Vibration in the present study does not refer to 
mechanical vibration induced by systems components, but rather vibration induced by environmental influences such as air turbulence. Vibration is most commonly quantified in terms of frequency, measured in cycles per second $(\mathrm{Hz})$, and amplitude, measured in meters $(\mathrm{m})$. Acceleration is measured in meters per second squared $\left(\mathrm{ms}^{-2}\right)$ or $\mathrm{g}^{\prime} \mathrm{s}(1 \mathrm{~g}=9.8$ $\mathrm{ms}^{-2}$ ). Because of the nature of sinusoidal vibration, root mean square (rms) acceleration is used to describe the magnitude of vibration. Vibration experienced during flight is almost never sinusoidal. Griffin (1990, p. 841) defines random vibration as "a vibration whose magnitude cannot be predicted precisely for any given instant in time." This definition gives a more realistic picture of the type of vibration pilots experience in a cockpit. Human performance under random vibration is not as easily measured as performance under sinusoidal vibration because for any given point in time the acceleration experienced will vary in an undefined manner. However, random vibration can be dealt with effectively by recording the actual acceleration level corresponding to each data point.

Measurements of vibration magnitude can be made separately on the $x, y$, and $z$ axes (Figure 1). Most experimental evaluations of vibration effects on manual skills are done using the vertical z-axis motion (McLeod \& Griffin, 1986) because this is the axis in which the greatest disruption in task performance is observed. Additionally, the z-axis is the one in which the most turbulence is experienced during flight, and therefore will be the axis of primary interest in the present stldy, however, acceleration levels for both the $y$ and $z$-axes will be collected. Acceleration on the $\mathrm{x}$-axis will not be measured in the present study because of the lack of additional equipment.

Human Response to Vibration. Vibrational effects on humans are studied in terms of whole-body and segmental vibration (local vibration). Whole-body vibration is defined as vibration which "occurs when the body is supported on a surface which is vibrating" 
Touchsorrans and Turbulence
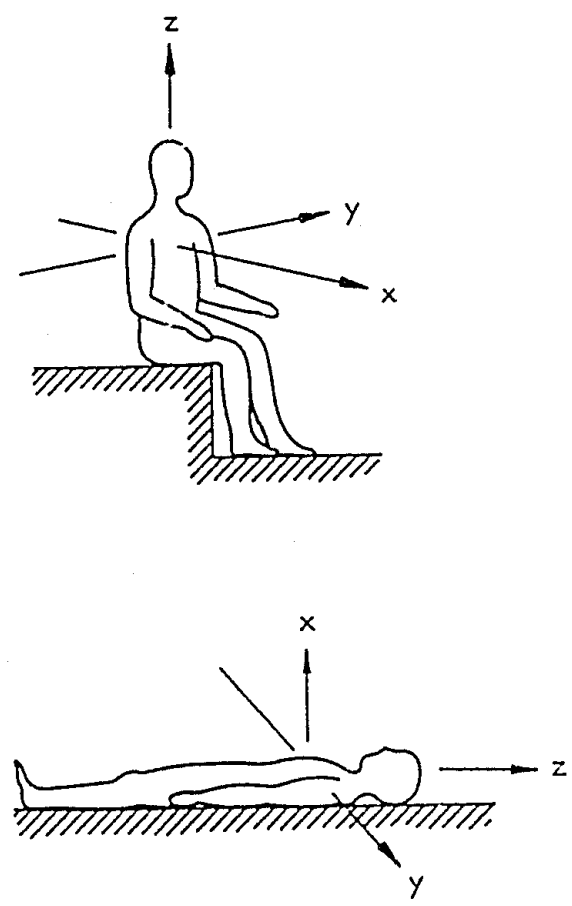

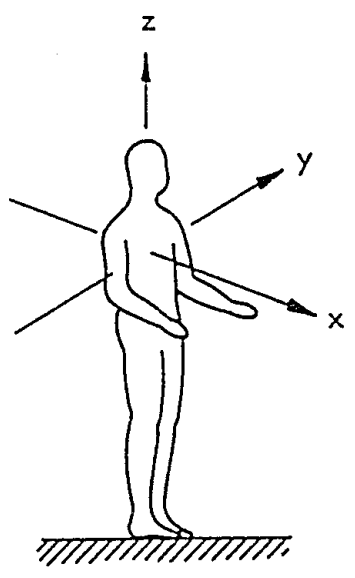

$$
\begin{aligned}
& x \text { axis - back-to-chest } \\
& y \text { axis - right-to-left side } \\
& z \text { axis - foot (or buttocks) } \\
& \text { to-head }
\end{aligned}
$$

Figure 1. X, Y, Z co-ordinate systenn (adopted from Griffin, 1990). 
Touchscreens and Turbulence

(Griffin 1990, p. 27). There are three principal ways in which vibration can enter the body causing whole-body vibration. These include sitting on a vibrating seat, standing on a vibrating floor, and lying on a vibrating bed. Locational vibration occurs when the head or one or more limbs are in contact with a vibrating surface (Griffin, 1990, chap. 2). Both whole-body and locational vibration can cause vibration to the entire body.

Vibration effects on seated subjects are directly applicable in the present study. Human response, in a seated position, to whole-body z-axis oscillations vary at different frequencies. At $2 \mathrm{~Hz}$, the parts of the body move up and down together accompanied with a sensation of being pushed up then floating down. During this motion, unsupported, free movements with the hand may be disturbed causing interference with hand positioning with the greatest interference occurring at around $5 \mathrm{~Hz}$ (Griffin, 1990, chap. 2). At higher frequencies, seated subjects will experience amplification of vibration within the body. At around $5 \mathrm{~Hz}$ the first major resonance occurs which accounts for the greatest amplification within the body. However, different parts of the body resonate at different frequencies, therefore making it difficult to precisely predict the vibration levels that will result in body resonance (Griffin, 1990, chap. 2).

The type of chair used and sitting posture are other factors which can greatly increase or decrease vibration experienced by a seated person depending on the frequency and direction of the vibration. Below 1-2 Hz, most seats have very little influence on perceived vibration. But at $4 \mathrm{~Hz}$, the vertical vibration is amplified, often doubling the acceleration. However, above $10 \mathrm{~Hz}$, a seat can attenuate vertical vibration thereby reducing the influence of the motion (Griffin, 1990, p. 31).

Another way in which vibration effects are measured involves the subjective responses of the operators to varying accelerations. A set of criteria is defined depending on the 
Touchscreens and Turbulence

effects the vibration has on comfort, activities, and health. From there, these criteria are assigned subjective (numerical) values associated with acceleration. According to Griffin (1990), the most commonly used criteria and their limits with a vibration frequency

between $1 \mathrm{~Hz}$ and $2 . \mathrm{Hz}$ are: fairly uncomfortable $\left(.5 \mathrm{~ms}^{-2}\right.$ or $\left..05 \mathrm{~g}\right)$, very uncomfortable $\left(1.25 \mathrm{~ms}^{-2}\right.$ or $\left..127 \mathrm{~g}\right)$, and extremely uncomfortable $\left(2.0 \mathrm{~ms}^{-2}\right.$ or $\left..204 \mathrm{~g}\right)$.

\section{Task Performance and Turbulence}

Task Definition. McLeod and Griffin (1986) classify manual control tasks into three types which are important when determining the magnitude of a vibration effect on the performance of a task.

Type A. Continuous tasks where the subject controls the hand freely in space as in pointing or reaching.

Type B. Continuous tasks where the subject's hand manipulates a control at a fixed position attached to the vibrating structure as in a joystick.

Type C. Tasks in which an operator performs a single, discrete operation as in pressing a button. Often times Type $C$ tasks are preceded by a Type $A$ task.

McLc _ and Griffin (1986) suggest evaluating Type C tasks separately from Type A tasks in instances where the former (C) follows the latter $(A)$. however, in the case of touchscreen manipulation in motion environments, an argument can be made for a new, fourth type of task, Type D.

Type D. A task where the successful execution of a Type $\mathrm{C}$ task is concurrent with the successful execution of a Type $A$ task, as in continuously touching a target on a touchscreen with no arm support or dragging a finger along a screen to select a desired target. 
Touchscreens and Turbulence

There are a number of mechanisms discussed by McLeod and Griffin (1986) which may mediate the disruption of a task during vibration. Vibration-correlated error is one mechanism which is directly applicable to the present study, and is defined as a motion or force at the hand that is linearly and directly correlated with the vibration input. The presence or absence of arm support is directly associated with the significance of vibrationcorrelated error. Experimental data collected by McLeod and Griffin (1986) has shown a disruption of type A and simple type B tasks to occur if acceleration magnitude exceeds around $.04 \mathrm{~g} \mathrm{rms}$. However, vibration-induced disruption of a manual task can occur as a result of any factor which initiates substantial changes in the transmission of vibration to the head, shoulder, or hand. These changes may also be a result of an operator's seating and arm supports.

Soine other mechanisms mediating vibration effects mentioned by McLeod and Griffin (1986) include interference with feedback loops within the neuro-muscular system and changes in an operator's perceptual-motor workload. McLeod and Griffin (1986) also mention that an increase in operator workload can impair performance especially when the operator is already performing close to the limits of their ability. Figure 2 shows a taxonomic model of human operator processes contributing to performance with vibration which has been adapted from Lewis and Griffin $(1976,1978)$ to include aviation cockpits with touchscreen interfaces. Lewis and Griffin (1976) theorized that it is possible to determine vibrational effects on various component processes of the human operator. The gross effects of vibration on an individual task could then be predicted by determining the contributions of the various component processes to total task performance. The effects are then integrated at the subsystem level. The model is designed as a tool to help identify possible weak points in a system or experimental design and correct for them. With the aid 
Touchscreens and Turbulence

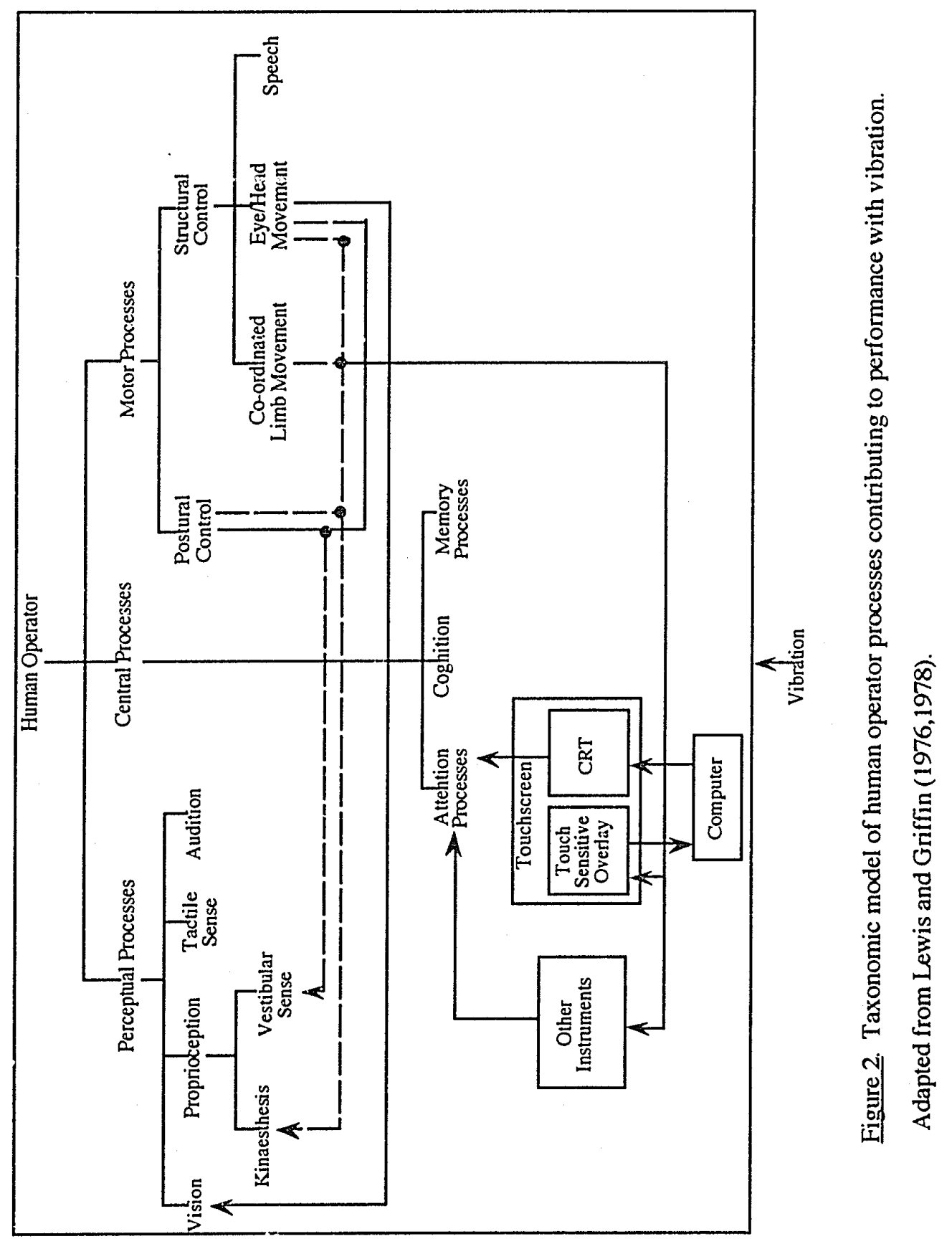


Touchscreens and Turbulence

of this model, it is possible to predict the performance effects of touchscreen use during vibration. As shown in the model, touchscreen use, in addition to other cockpit instruments, form a heavy load on a pilot's attentional processes which consequently generates an increase in workload. Additionally, touch sensitive overlays require the use of coordinated limb movements and are indirectly related to other perceptual, central, and motor processes. An increase in cockpit workload in the form of an attentional input could directly affect the perceptual and motor processes involved with touchscreen interaction, thereby confounding any direct effects vibration might have on operator performance using touchscreens. To the extent that Lewis and Griffin's (1976) model is transferable to the present situation, the degree to which proprioception is disrupted due to turbulence could determine the amount of performance decrement experienced while using the touchscreens.

Using the literature on manual control and vibration tasks along with touchscreen characteristics, three levels of turbulence were selected to determine its effect on operator touchscreen use. The first level consisted of no turbulence in order to establish a baseline for performance effects. As mentioned previously, the disruption of type A and B tasks is first observed at around $.04 \mathrm{~g} \mathrm{rms}$. Therefore the second turbulence level corresponded to this acceleration value in order to determine a minimum threshold of error tolerance. Turbulence level three, $.055 \mathrm{~g} \mathrm{rms}$, is the maximum acceleration the simulator is presently capable of generating and was used to help determine the maximum threshold of error tolerance. This level roughly corresponds to a subjective semantic label of "extreme turbulence" as defined by the Airman's Information Manual (AIM) in their turbulence intensity criteria table. Extreme turbulence is defined by the AIM as the point at which the "airplane is tossed violently about; practically impossible to control, may cause structural damage" (AIM, 1988). 
Touchscreens and Turbulence

\section{Objective}

The present study was intended as a preliminary investigation into the effects of turbulence while using two forms of activation methods to interact with touchscreens in a very specific operating environment. Two levels of turbulence (medium and high) with a control condition (no turbulence) were used and the amount of finger positioning error generated during corresponding acceleration was measured. The goal was to determine what factors influence finger movement on the screen, and which activation method was the most effective method in terms of speed and accuracy. It is difficult to make predictions about the performance of the activation methods based on the current literature; in addition only speculative predictions can be made into human response using touchscreens during turbulence. The results of this study should aid in the future formulation of more meaningful hypotheses regarding human performance using touchscreens in motion environments.

Method

\section{Subjects}

Sixteen crews of pilots from a major U.S. airline were used for a total of 32 subjects. Each crew consisted of a Captain and a First Officer, and was recruited and paid ihrough Bionetics Corporation. All subjects were required to hold a current rating in a glass cockpit aircraft and subjects were assigned to their seat position (Captain, First Officer) depending on their current seat position with their present airline. Because planes differ in their cockpit technology, pilots were paired based on the type of plane they fly in order to minimize discrepancies which may arise due to these technological differences. Subjects were treated in accordance with the ethical standards and guidelines employed by 
Touchscreens and Turbulence

NASA/Ames Research Center and the San Jose State University Institutional Review

Board.

Apparatus

Simulator. The Advanced Concepts Flight Simulator (ACFS) located at NASA-Ames Research Center was used for this experiment. The ACFS is part of the Man-Vehicle Systems Research Facility (MVSRF) and was developed jointly by NASA and the Lockheed-Georgia Company. The ACFS is a generic representation of present and future aircraft designs with a flight station designed for a two-member crew. All controls necessary for aircraft operation are easily accessible by both pilots (Figure 3). The flight station contains a desktop surface located over the pilots' knees which provides arm and elbow support. Five vertically mounted, 13-inch raster-generated, full color shadowmasked CRT displays make up the main instrument panel. The three middle CRT displays are equipped with resistive touch panels. Figure 4 shows the anthropometric relationship between the pilots and the CRT displays in the cockpit.

Personnel. An experiment operator, an air traffic controller (ATC), and a "pseudo" pilot were used to create a realistic flight environment. The experiment operator was responsible for all normal company ground, maintenance, dispatch, and flight attendant communications. ATC provided weather information, clearances, ground, tower, departure, approach, and center communications. The "pseudo" pilot used a voice disguiser to provide "traffic" or "pseudo" aircraft communications.

Computers. The simulation was driven by a DEC VAX 8830 computer. The flight displays were driven by three 4D-20 and one HD 80-GT Silicon Graphics IRIS workstations. A VAX 6310 services the Air Traffic Control facility which works in conjunction with the simulator. 
Touchscreens and Turbulence

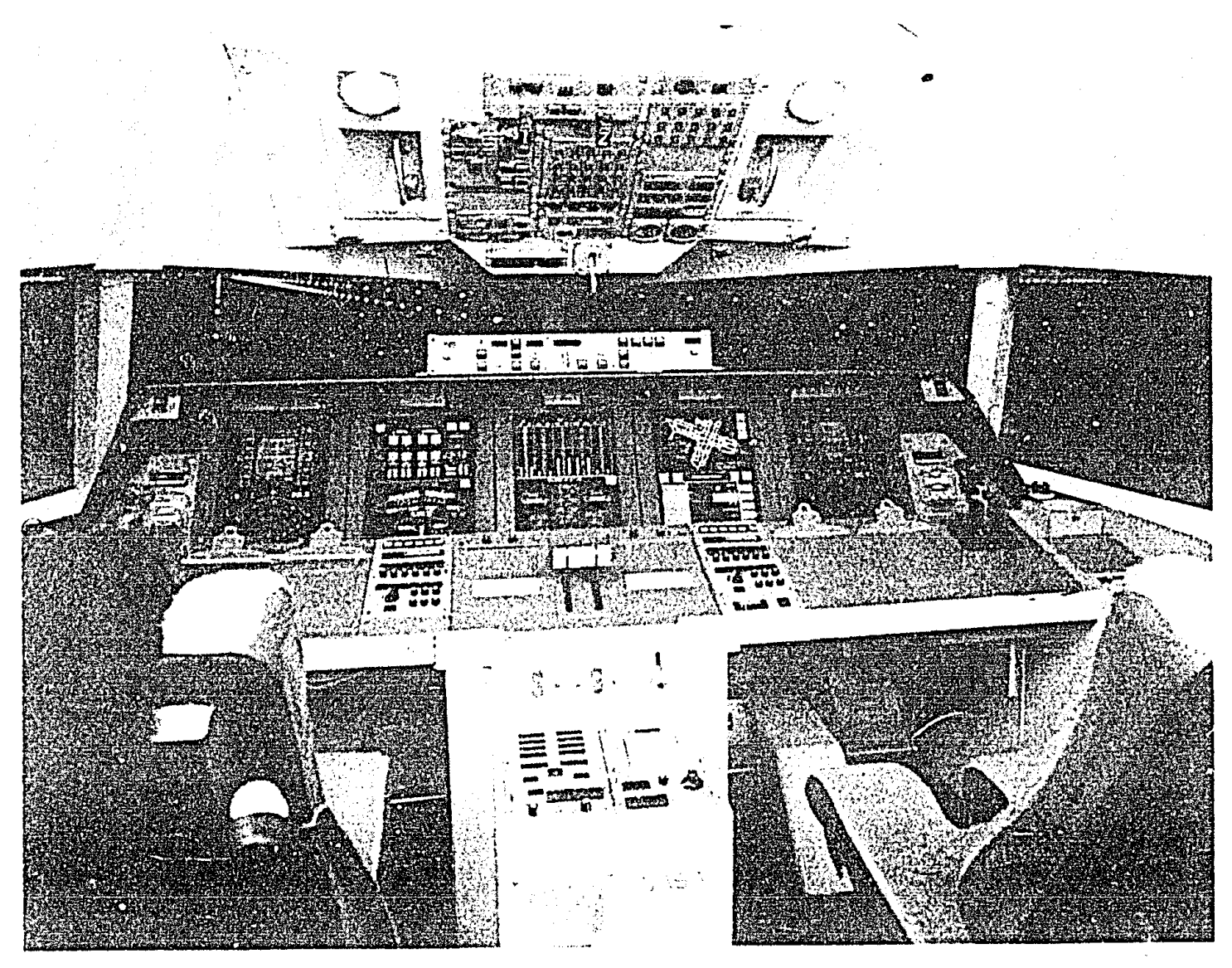

Figure 3. View of the Advanced Concepts Flight Simulator. 
Touchscreens and Turbulence

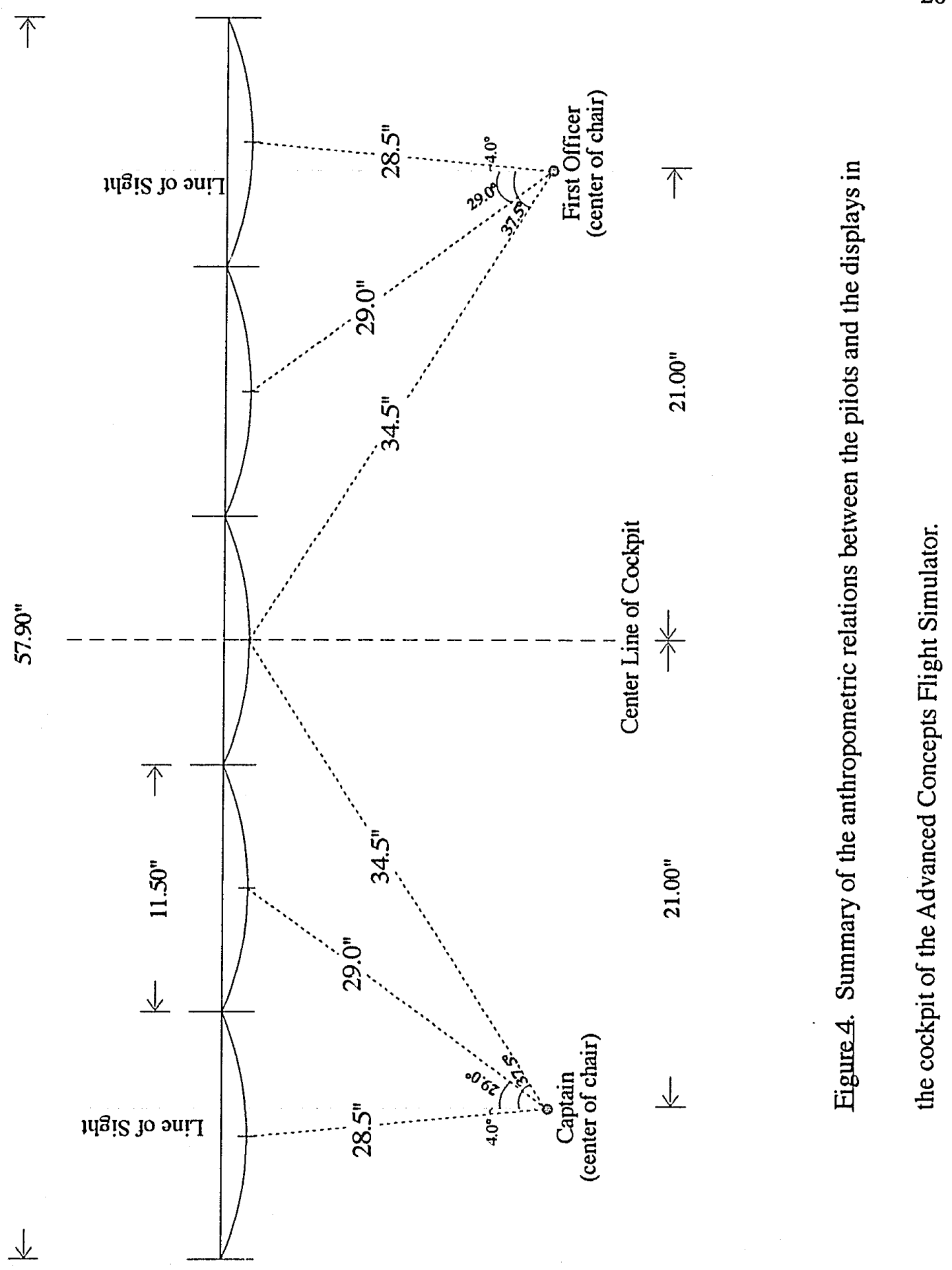


External visual scenes were provided by a computer-generated visual system called Image III, which is built by the Link-Miles Company of England. This system provides color scenes representing the outside world which depict specific airports and their surroundings as viewed at dusk or night from the cockpit (Chappell \& Sexton, 1986; James, 1987).

Touchscreens. The touchscreen overlays were Transparent Device model number E274-13HSC, resistive, flat panel overlays. They consist of twio layers of transparent material with 25 rows and 40 columns of clear separator dots. An actuation force of 30 grams is required to register a touch. The panels are designed to be placed over a 13-inch CRT display. The active area of the panels measures 8 inch $\times 11$ inch. The panel contains 7.27 touchcells per inch along the vertical dimension and 6.25 touchcells per inch along the horizontal dimension, for a total of 4000 touch points. Figure 5 is an example of a touchcell grid with $\mathrm{x}, \mathrm{y}$ dimensions.

Primary touch inputs were in the form of button pushes. The $\mathrm{x}$ and $\mathrm{y}$ finger position coordinates for the duration of pilot interaction with the touchscreens were collected. Checklist use will provide the bulk of the button touches. Pilots use the checklist buttons to "maneuver" through a series of items which prepare the airplane for the next phase of flight. Figure 6 shows the checklist button and its corresponding measurements. Although checklist buttons appear to be continuous, active areas only reside on the left and right ends of the button. Active areas are indicated by grey shaded boxes. The active area on the left, or the title side, measures .55 inches by 2.0 inches. The active area on the right, the response side, measures .77 inches by 2.0 inches. The response area is larger because it is the more frequently touched area of the checklist button. 
Touchscreens and Turbulence

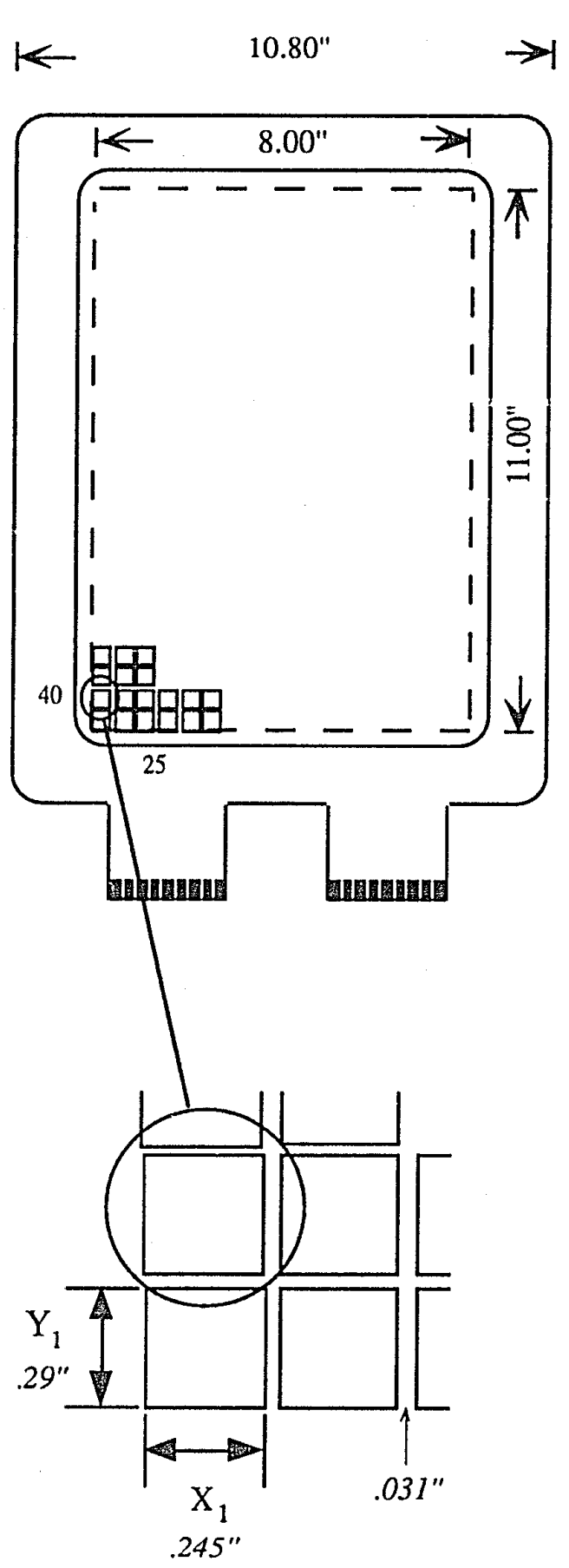

Figure 5. Touchscreen measurments along with touchcell grid and $x, y$ dimensions. 
Touchscreens and Turbulence

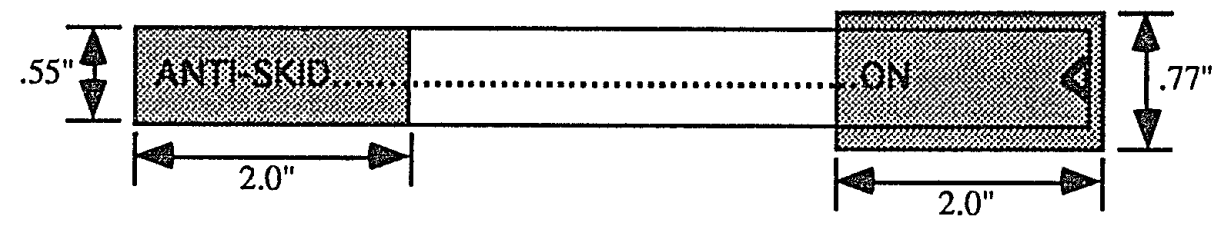

Figure 6. An example of a checklist button, grey areas define active areas. 
Touchscreens and Turbulence

Design

The experimental design was a $2 \times 3$ mixed factorial design with repeated measures. The independent measures of interest were "First Contact" and "Lift-Off" activation methods and three levels of turbulence, none, medium (.035 $\mathrm{g} \mathrm{rms})$, and high (.055 $\mathrm{g}$ rms). Activation method was a between subject variable and turbulence was a within subjects variable. Subjects performed three complete replications (3 legs) of the design. Turbulence levels were counterbalanced across the three legs such that each level followed every other level an equal number of times.

\section{Procedure}

Each leg on a mission was divided into different phases of flight. These phases consist of takeoff/climb, cruise, and descent/landing. During each phase, with the exception of cruise, pilots were required to complete one or two checklists. The button presses from the use of these checklists provide the data used in this study. The cruise phase of flight does not require checklist interaction, so a simple aircraft malfunction was generated in order to elicit checklist use. Because of the potential impact of workload on pilot performance, these malfunctions were specifically chosen for their low workload.

Pilots experienced three levels of turbulence during each leg with the turbulence levels being counterbalanced across legs. The three levels of vibration were: no vibration $(0 \mathrm{~g}$ $\mathrm{rms})$, medium vibration (.035 $\mathrm{g} \mathrm{rms}$ ) and high vibration (.055 $\mathrm{g} \mathrm{rms})$. However, because of the randomness of the vibration, pilots experienced a range of turbulence around the means stated above. The minimum and maximum acceleration ranges for the medium and high vibration conditions are $-.12 \mathrm{~g}$ to $.13 \mathrm{~g}$ and $-.18 \mathrm{~g}$ to $.18 \mathrm{~g}$ respectively.

A problem arises when either medium or high vibration is present in the descent and landing phases of flight. Airline procedures call for a go-around when turbulence is too 
Touchscreens and Turbulence

severe to stabilize the plane for landing. Both the medium and high turbulence conditions cause this problem. By gradually lowering the turbulence level back down to zero at around 3,000 feet, this problem is alleviated. However, in doing so, the landing checklist is excluded from data collection thereby greatly decreasing the number of button touches collected under the last turbulence condition. To correct for this, an additional malfunction was added to all legs in the last phase of flight. Figure 7 shows each leg along with the corresponding checklist/malfunction and the approximate number of button touches to be collected. The number of touches varied by a few touches because there are several different ways in which pilots can page through the system and this introduced a small variability in the total number of button presses collected for each crew.

Each crew took two days to perform the experiment. The morning of the first day was ground school, with the afternoon being spent in the simulator with an instructor actually flying the plane. The morning of the second day was a training session, and the afternoon was the actual experiment run. Subjective data was collected through a series of debriefing questions collected at the termination of the experiment.

Training. A total of eleven hours were spent familiarizing and training the pilots. Subjects were instructed not to use anything other than their fingers to touch the screens.

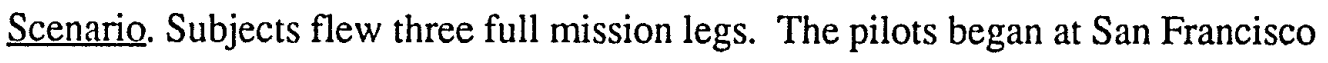
International Airport (SFO) and flew to Sacramento Airport (SMF), then on to Stockton Airport (STK) and finally ended back at San Francisco Airport.

SFO - SMF. When the wheels of the airplane lift off the ground, the first turbulence condition was activated. Forty-five seconds after the last item on the After Takeoff checklist was completed the second turbulence condition began. One second after the second turbulence condition began, the \#1 line contactor opened. Forty-five seconds after 
Touchscreens and Turbulence

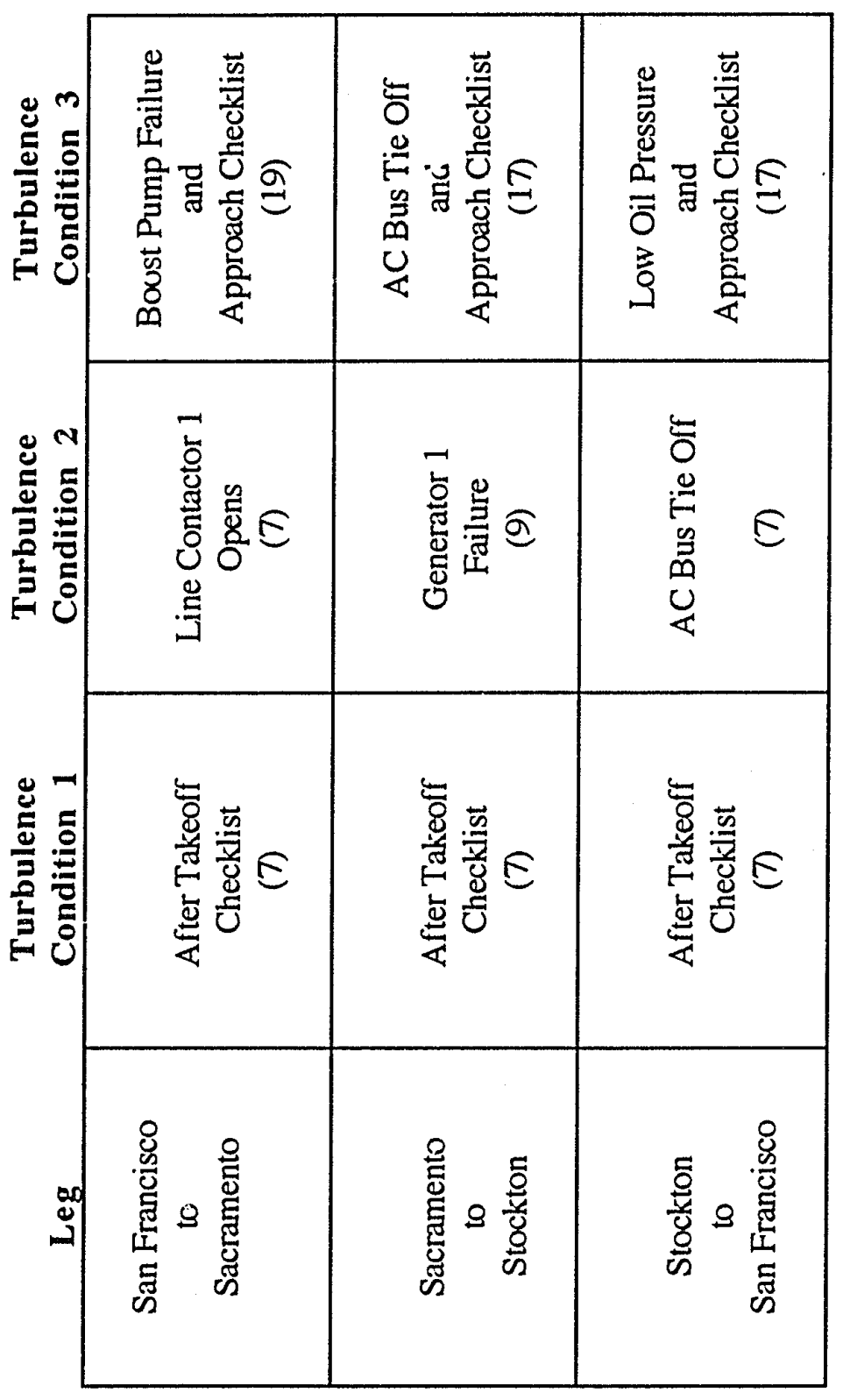

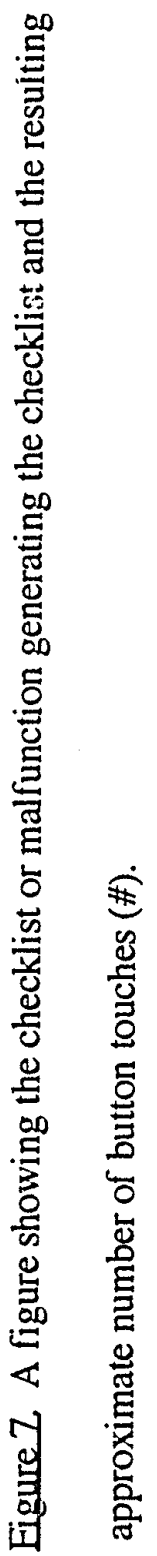


Touchscreens and Turbulence

the completion of the Line Contactor Off checklist, the third turbulence condition was activated. One second after the onset of the third turbulence, a boost pump failure occurred which called for the AC Bus Tie Off checklist. Following the completion of the irregular checklist, the Approach checklist was completed, after which the turbulence began $t$. decline back down to zero for landing.

SMF - STK. This scenario was the same as the previous leg, including the timing, except the systems malfunction during the cruise phase 0. flight was a generator \#1 failure requiring the Gen Failure checklist. An AC Bus Tie off occurred during the last turbulence condition and required the AC Bus Tie Off checklist, followed by the Approach checklist.

STK - SFO. The scenario was the same as the previous two legs except that the $A C$ Bus Tie Off malfunction occurred during the cruise phase of flight, requiring the same $A C$ Bus Tie Off utilized in the previous legs. Additionally, the last turbulence condition included a low oil pressure malfunction which required the Low Oil Pressure checklist followed by the Approach checklist.

\section{Results}

Data was collected for each finger touch on the screen in the form of $x, y$ finger coordinates at a sampling rate of $30 \mathrm{~Hz}$. In other words, the $x, y$ finger position was measured from the time the finger touched the screen to the time it was lifted off for both activation methods. To account for the randomness of the vibration, acceleration levels were collected with a sampling rate of $30 \mathrm{~Hz}$ to coincide with the $\mathrm{x}, \mathrm{y}$ coordinates. This information was obtained for the $z$ and $y$ axes from accelerometers located under each pilot's seat. The values obtained for each $\mathrm{z}$ and $\mathrm{y}$ axis were averaged to obtain a mean $\mathrm{rms} \mathrm{z}$ (MEANRMSZ) and a mean rms y (MEANRMSY) value for each continuous touch. Other 
data obtained included measures of arm support, determined by the support received by the elbow and/or wrist at the time of the touch.

\section{Dependent Measures}

Each individual touch was characterized using three separate methods: touch duration, path length, and ADM (Average Distance from Mean). The first measure used was a reaction time measure recording the amount of time in which the subject's finger was in contact with the screen. The time recorded at the beginning of the touch is subtracted from the time recorded at the end. The second dependent measure developed was a path length measure. While the subject's hand was in contact with the screen, it often moved. Causes for this motion may come from voluntary action, such as correction of a missed touch, or involuntary action, such as turbulence. The path length is the actual distance traveled by the subject's finger while in contact with the screen and can be characterized by the following equation:

$$
\text { Path Length }=\sum_{i=1}^{\mathrm{n}-1} \sqrt{\left(\mathrm{x}_{i+1}-\mathrm{x}_{i}\right)^{2}+\left(\mathrm{y}_{i+1}-\mathrm{y}_{i}\right)^{2}}
$$

In most cases this is not a linear path but rather representative of a more circular path. The third measure developed is termed the average deviation from the mean (ADM) and was derived using the following equation:

$$
\operatorname{ADM}=\frac{\sum_{i=1}^{n} \sqrt{\left(x_{i}-\bar{x}\right)^{2}+\left(y_{i}-\bar{y}\right)^{2}}}{n} .
$$

The measure of finger duration will primarily be used to evaluate the activation methods. Both ADM and path length will be used to evaluate turbulence effects along with activation methods. The goal in the analyses will be to determine what factors influence finger 
movement on the screen, and which activation method was the most effective method in terms of speed and accuracy.

\section{Analyses}

A summary of the descriptive data is displayed in Table 1. No main effects of repetition (legs) were found, and consequently, the data were collapsed across legs. Separate 2 (activation method) $\times 3$ (turbulence levels) x 16 (subjects) mixed analyses of variance were performed for the dependent variables of duration, ADM and path length. Duration. A main effect of activation method on duration was found $F(1,14)=4.26$, $p<.058 ; \hat{w}^{2}=.16$ (Figure 8). The finger duration on the screen was longer for the lift-off method of activation than for first-contact. An interaction between activation and turbulence was found $F(2,28)=3.27, p<.053 ; \hat{w}^{2}=.12$ (Figure 9). An additional oneway analysis of variance for touch duration was performed on activation method excluding any touches longer than one second. The rationale behind this analysis followed the observation that some subjects tended to hold their finger on the screen for long periods of time while performing other tasks. Clearly, these touches were not a result of the differences between activation methods, but rather individual idiosyncrasies. The results showed a significant main effect of activation method on duration $F(1,2343)=77.21$, $p<.001$.

ADM. No main effects or interactions were found for ADM.

Path Length. A main effect of turbulence on path length was found $F(2,28)=3.56$, $p<.05$ (Figure 10). No other main effects or interactions were found for path length.

Multiple Regression. Three stepwise multiple regression analyses were performed on the data in an attempt to determine which factors, aside from activation method and 
Touchscreens and Turbulence

Table 1

Descriptive statistics

\begin{tabular}{lcccc} 
Variable & Mean & Std Dev & Min & Max \\
& & & & \\
\hline Mean Rms Z (g) & .03 & .02 & .00 & .11 \\
Mean Rms Y (g) & .01 & .01 & .00 & .09 \\
ADM & .71 & 1.02 & 0 & 12 \\
Path Length & 3.62 & 5.10 & 0 & 45 \\
Duration (sec) & .46 & 1.38 & 0 & 42 \\
\end{tabular}


Touchscreens and Turbulence

31

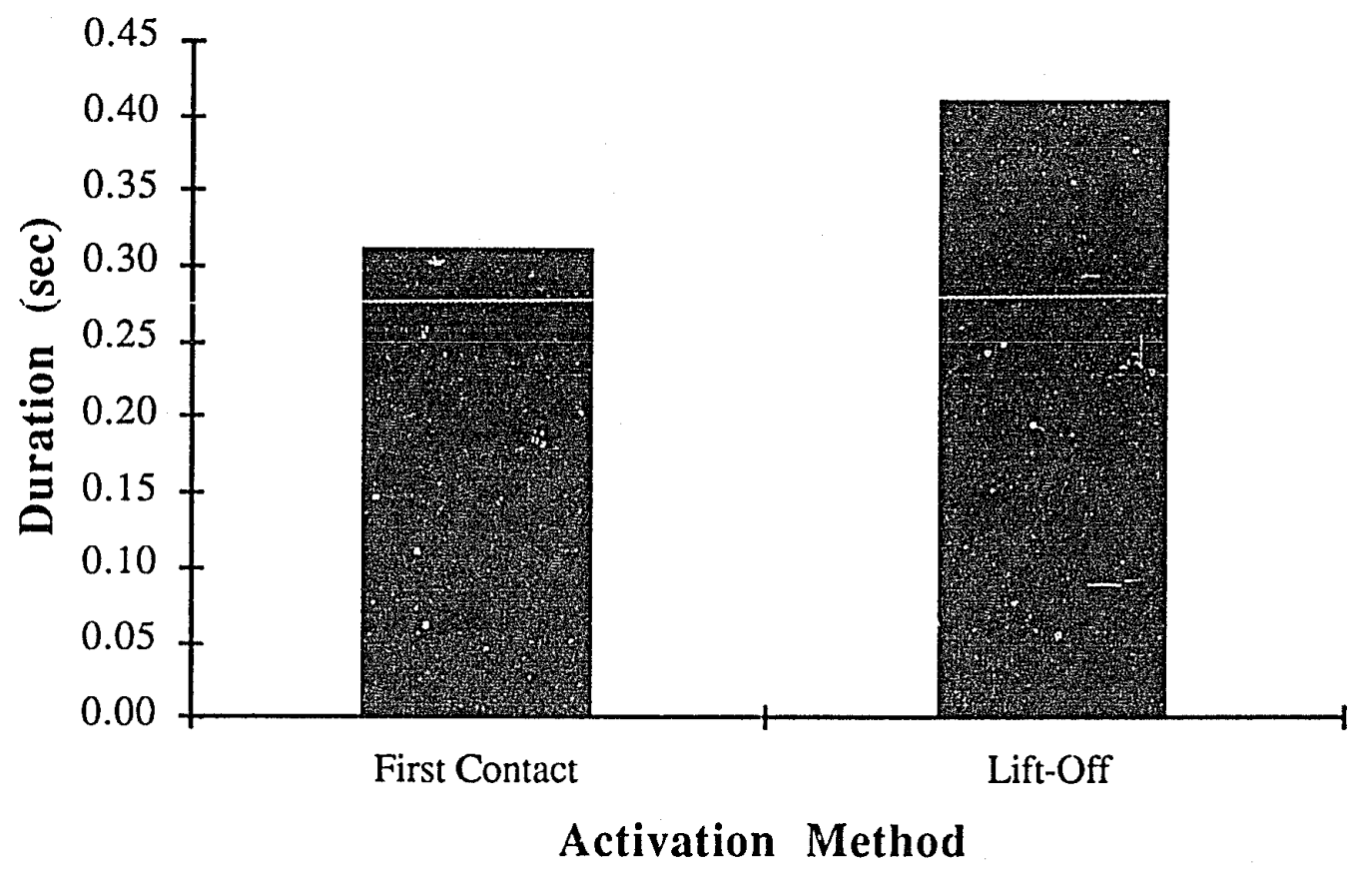

Figure 8. Main effect of activation method on duration. 
Touchscreens and Turbulence

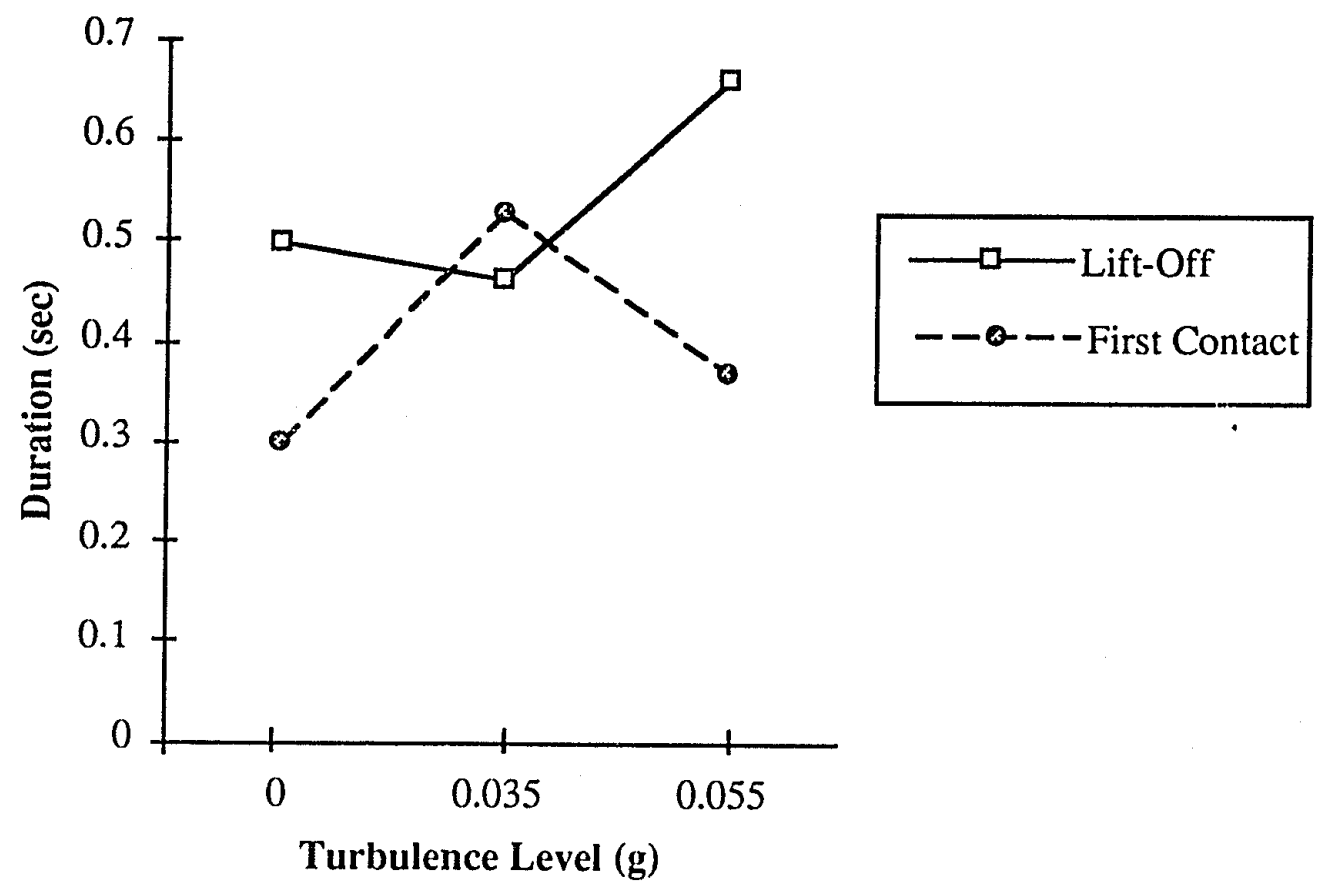

Figure 9. Interaction between activation method and turbulence for duration. 
Touchscreens and Turbulence

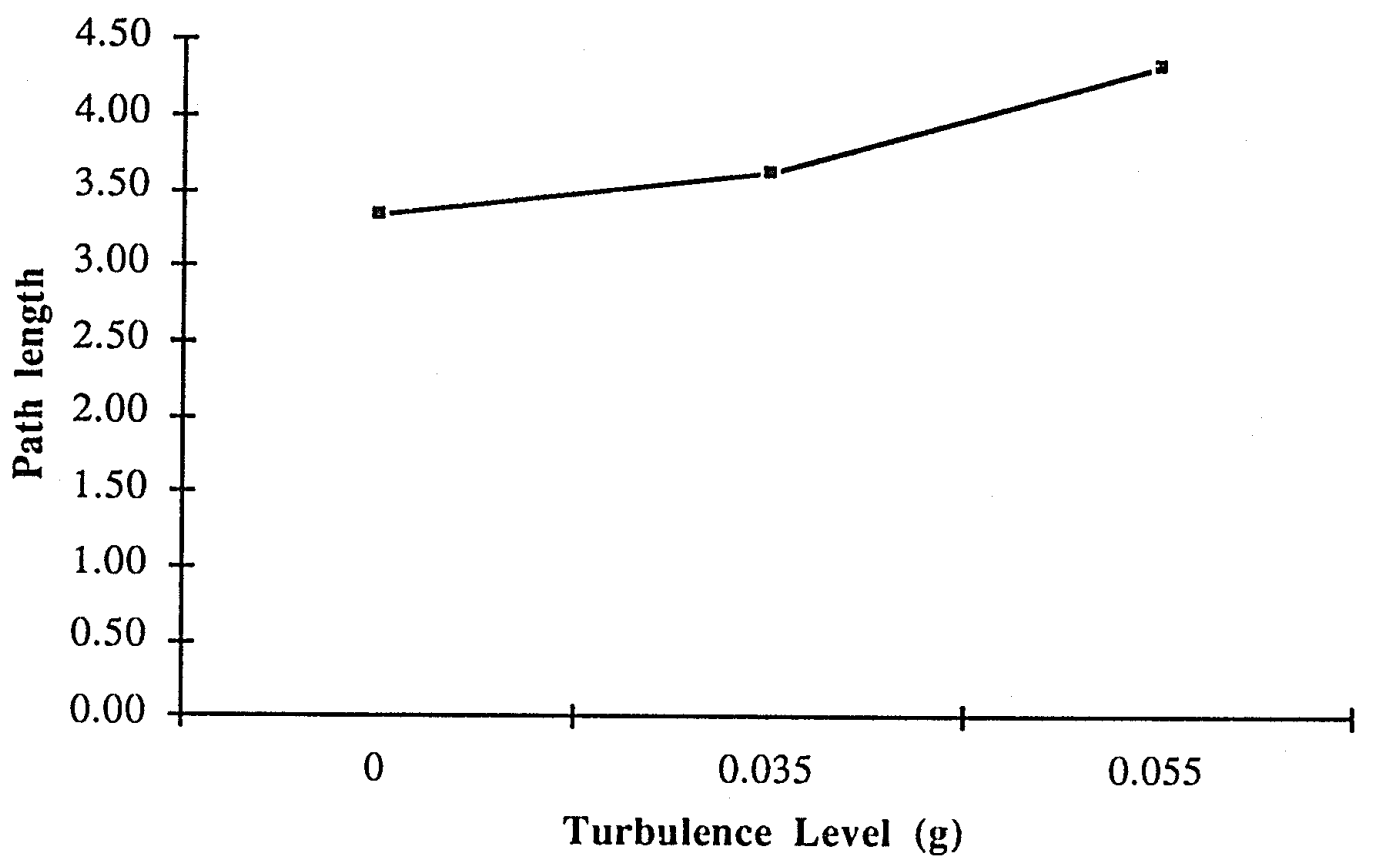

Figure 10. Main effect of turbulence on path length. 
Touchscreens and Turbulence

turbulence levels, contributed most to the variance and which variables are more important predictors for the three dependent measures of duration, ADM, and path length. Along with activation method and turbulence, crew, elbow support information, wrist support information, mean rms z-axis, and mean rms y-axis information were entered into the regression. Table 2 shows the correlation matrix for the data. Table 3 shows the results of the multiple regression analysis with the relative Beta weights and $F(E g n)$ values.

\section{Discussion}

\section{Activation Method}

Lift-off method of activation had a significantly longer input time than first contact. This directly supports the findings of Potter et al. (1988), and displays the inherent differences between the two types of activation methods. With the lift-off strategy, a few more milliseconds are required for the subject to lift off the screen, whereas the first contact method activates a target the moment the finger touches an active area. The regression analysis performed on duration to determine significant predictors revealed activation method as the only significant predictor of duration. This provided further confirmation that the variance found in duration is due to the different activation methods. However, only $0.23 \%\left(\mathrm{R}^{2}=.0023\right)$ of the total variance in duration was accounted for by activation method. This would seem to indicate that there are other factors contributing to the variance in duration which were either not entered into the present regression analysis, or not measured at all.

An interaction between turbulence level and activation method was found for duration which suggests that there is a trade-off between the two types of activation methods during turbulence, especially during medium levels of turbulence of $.033 \mathrm{~g} \mathrm{rms}$ (Figure 9). However, at this time it is difficult to give an explanation of this interaction without further 
Touchscreens and Turbulence

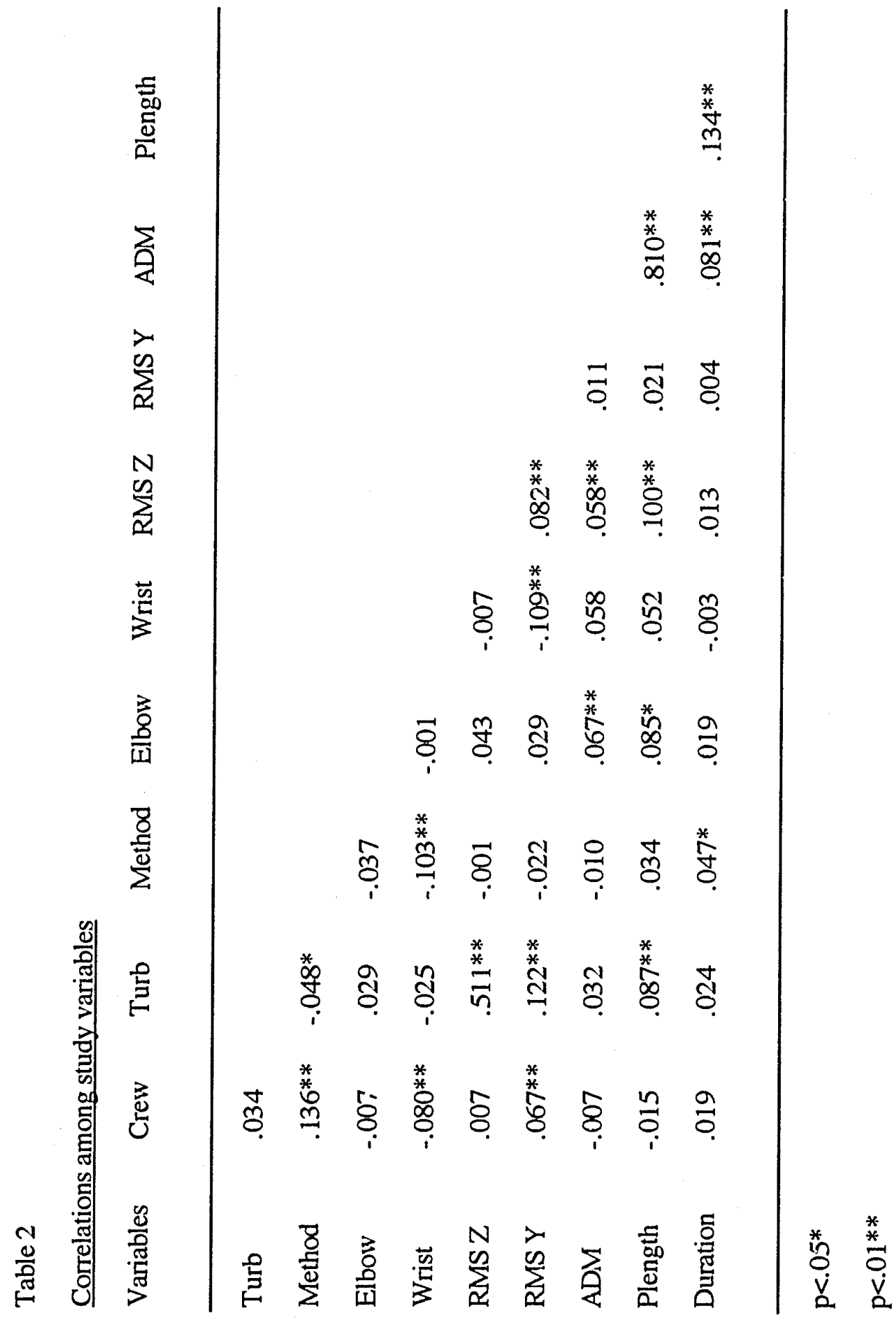


Table 3

Variables which entered into the regression analyses.

\begin{tabular}{lll} 
Step & Beta & F \\
\hline
\end{tabular}

Dependent variable: Duration
1. Method
.048
$5.509 * *$

Dependent variable: ADM

$\begin{array}{lccc}\text { 1. } & \text { Mean RMS Y } & .155 & 87.328 * * \\ \text { 2. } & \text { Mean RMS Z } & .109 & 57.994^{* *} \\ \text { 3. } & \text { Wrist } & .066 & 42.522 * * \\ \text { 4. } & \text { Elbow } & .057 & 34.033^{* *}\end{array}$

Dependent variable: Path Length

$\begin{array}{lccc}\text { 1. } & \text { Mean RMS Y } & .196 & 169.942^{* *} \\ \text { 2. } & \text { Mean RMS Z } & .201 & 126.209^{* *} \\ \text { 3. } & \text { Wrist } & .059 & 87.211^{* *} \\ \text { 4. } & \text { Turb } & -.044 & 66.654^{* *} \\ \text { 5. } & \text { Elbow } & .039 & 54.167 * * \\ \text { 6. } & \text { Method } & .039 & 45.881^{* *}\end{array}$


study. Data gathered from the video tapes revealed that subjects tend to rest their finger on the screen longer while using the lift-off activation method. This suggests that subjects felt more comfortable keeping their finger on the screen for long periods of time with the liftoff activation method without risk of an error occurring even while experiencing turbulence.

Turbulence

Some difficulty was encountered when determining the appropriate means by which to characterize the data. At the onset, the root mean square error (RMSE) was to be calculated based on the assumption that subjects would miss the target altogether during severe turbulence. The RMSE would then be calculated based on the centroid of the target. However, subjects did not touch outside the button despite the presence of turbulence. It also became clear that basing measurement on the centroid of the button was unacceptable, since this assumed that subjects intended to touch the precise center of the button. This holds especially true when interacting with checklist buttons due to the dimensions of the button (Figure 6). The subjects may have been more concerned with centering vertically as a result of hand/finger displacement due to turbulence, and less concerned about horizontal alignment. Video tapes revealed that subjects touched the area of the button closest to the resting place of their finger at the time the touch was initiated. Since no subjects touched outside the intended button, it was more reasonable to assume that the intended center of the touch was the mean of the collected $x, y$ coordinates for the touch. From there, ADM was calculated. It was also apparent that a measure which characterized the ccmplete movement of the finger was necessary. Therefore, path length was chosen to represent the entire path the finger traced on the screen. These two measures aid in achieving perhaps 
Touchscreens and Turbulence

the most important goal of this study which was to determine turbulence effects on finger motion on the screen.

The results of the analyses indicated that path length was a more characteristic measure of finger movement on the screen experienced during this experiment than ADM. Subject's path length increased as turbulence levels increased. This is a predictable response based on vibration-correlated error which is linearly and directly correlated with the vibration input (Griffin, 1990; McLeod and Griffin,1986). The resulting finger movements from the turbulence were not gross, large finger movements, but rather more fine movements such as the wobbling of a finger tip. As a result, the average deviation from the mean was not a very effective measure in this situation because the finger did not deviate far from the center of the touch.

The regression analysis showed that mean rms $z$, elbow, wrist, turbulence, and activation method are all significant predictors of path length with all predictors accounting for $2.3 \%$ of the total variance in path length (Table 3 ). Of all the predictors, mean $\mathrm{rms} z$ alone accounted for most of the variance with an $\mathrm{R}^{2}$ of .01 . This result confirms McLeod and Griffin's (1986) findings that the greatest amount of performance disruption occurs on the z-axis. Additionally, because the simulator was designed to mimic real turbulence, the relative magnitude of turbulence on the $z$-axis was greater then $y$-axis turbulence. This might explain why turbulence on the $y$-axis was not a significant predictor of path length. The amount of turbulence experienced on the z-axis is positively correlated with the path length of the finger on the screen. Only a very small amount to variance in path length is accounted for by the entered variables which would suggest that there are other variables which might account for more of the variance that were either not measured or entered into the regression analysis. 
Touchscreens and Turbulence

Arm Support

Elbow and wrist support are both significant predictors of path length; path length increases when the arm is supported. This is somewhat counterintuitive in that one would expect arm support to attenuate the amount of movement the finger experienced on the screen due to vibration. However, in the present situation, it is very likely that the desktop surface where the elbow and wrist were supported acted to accentuate finger motion. This is similar to the way in which backrests can work to both attenuate or provide additional input into the body, giving rise to greater vibration (Griffin, 1990, chap. 8).

\section{Subjective Data}

Slightly over half of the subjective reports reveal that turbulence experienced in the simulator used for this experiment was not very representative of actual turbulence encountered on the line. Most pilots felt that the turbulence they experienced in the simulator was lacking in the "g-loads" they usually experience. The amplitude of the turbulence was not sufficient to simulate the "drops" experienced during flight . Turbulence experienced on the line varies more in intensity and severity while the turbulence in the simulator was more mechanical and bumpy, "like driving in a $4 \times 4$ ". About a third of the subjects felt that, for the most part, the turbulence accurately represented that which is felt on the job. However, real turbulence is not as continuous as what they experienced on the simulator, and this type of continuous turbulence is more like "light chop" rather than moderate to severe turbulence. Figures 11 and 12 show subjective responses to two debriefing questions dealing with the relative turbulence experienced in the simulator and on the line and the difficulty of using touchscreens during turbulence. 
Touchscreens and Turbulence

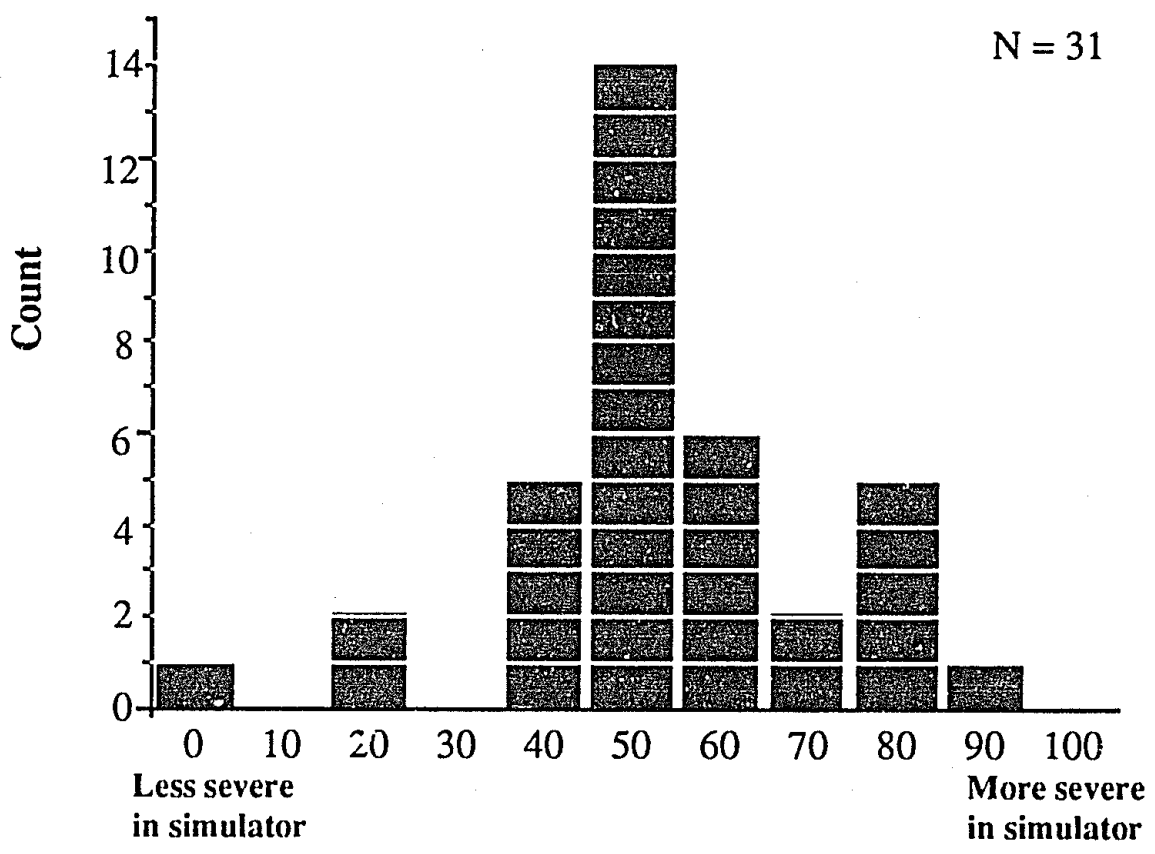

Figure 11. How did the severity of turbulence in the simulator compare to turbulence you experience while flying on line? $(\mathrm{N}=31$, one $\mathrm{n} / \mathrm{a})$ 


\section{Touchscreens and Turbulence}

41

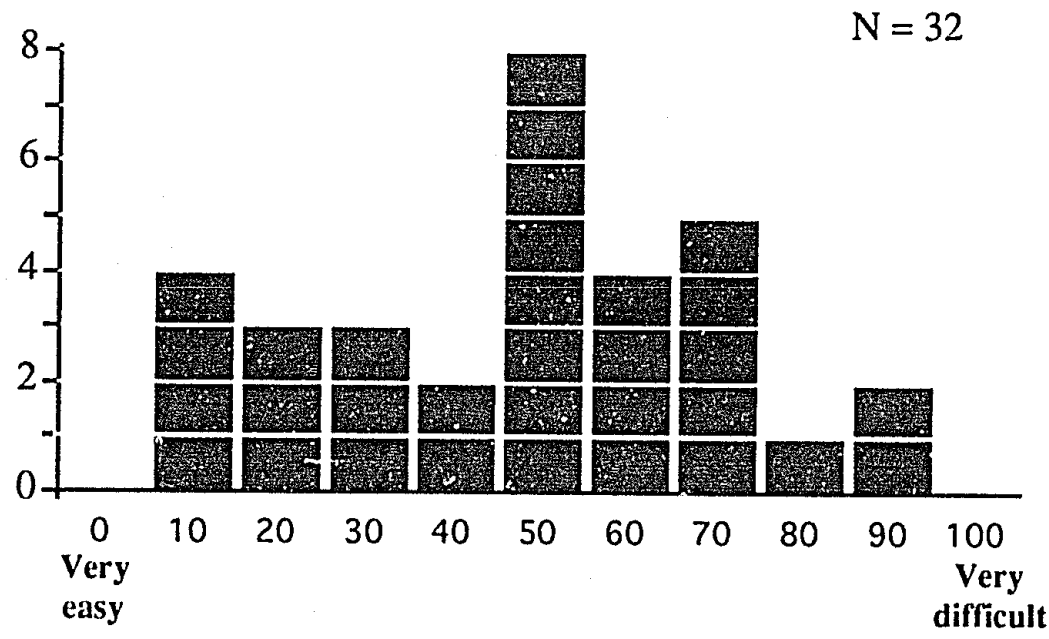

Figure 12. How difficult was it to use the touchscreens during turbulence in the simulator? 
Touchscreens and Turbulence

Conclusion

The present study is intended as a preliminary investigation into the effects of turbulence while using two forms of activation methods to interact with touchscreens in a very specific operating environment. The goal was to determine what factors influence finger movement on the screen, and which activation method was the most effective method in terms of speed and accuracy.

Apparently, there are other factors playing a role in the effectiveness of one activation method over another as evidenced by the small proportion of variance in duration accounted for by activation method. Those variables which may account for some of the variance may be target size, reach distance, or screen placement. In addition, some form of error measure might prove useful in evaluating differences between activation methods during turbulence. These error measures might include measuring false lift-offs, the inadvertent removal of the finger from the screen, or false land-ons which are erroneous touches to the screen as a result of a sensitive touchscreen (Sears et al. 1990). Measures of error associated with aviation environments might also be appropriate but are more difficult to define and quantify and are usually specific to the environment specific (Billings, Wick, Gerke \& Chase, 1971; Ruffel Smith, 1979).

Although a main effect of turbulence was found on path length, the levels of turbulence experienced during the present experiment resulted in no errors. Our measured effects were on very fine movements. If the task being performed requires "pixel" accuracy or very small targets, than turbulence of this nature might be a concern, but for the purposes of the present situation, it was not a hindrance. However, one must take into account the pilots' subjective responses to the turbulence, which reveals that the turbulence experienced was not very characteristic of what is felt while on the job. Additionally, based on the 
Touchscreens and Turbulence

43

results of the regression, there may be other factors which may be significant predictors of finger movement on the screen. These predictors might include target size and handedness.

Future Studies. The present study was intended as a preliminary look into the effects of turbulence on an operator's use of touchscreens in an aviation environment. Future researchers wishing to study the effects of turbulence on a subject's use of touchscreens would do well to use more severe turbulence in order to actually get at some type of threshold measurement of error tolerance. Additionally, more research into the effect of variables such as interface design, arm support, and handedness would prove beneficial. Although many of these factors have already been studied in the context of touchscreen use, they have been performed in controlled laboratory environments. Re-evaluation of factors affecting touchscreen use needs to be taken out of the laboratory and placed in an environment where potential users perform actual tasks in a realistic environment. Only then will we begin to understand the type of effects dynamic factors, such as turbulence, have on the performance of touchscreen users. Further investigation into the effect of turbulence on pilot performance in the cockpit is warranted before touchscreen technology can be implemented effectively into airplane cockpits. The results of the present study should not be used as a guideline to implementing touchscreen technology into cockpits, but rather as a guideline to the direction future research should take. 
Touchscreens and Turbulence

References

Beringer, D. B., \& Lee, K. M. (1988). Parameters influencing the airborne application of touch devices for data entry and retrieval: Phase 1: Technological, positional, and chronological effects (Technical Report MDHC-88-1/HFRL-88-2). Las Cruces, NM: Human Factors Research Laboratory, New Mexico State University.

Billings, C. E., Wick, R. L., Jr., Gerke, R. J., \& Chase, R. C. (1971). The effects of alcohol on pilot performance during instrument flight (Project RF-2626). Columbus, Ohio: The Ohio State University Research Foundation.

Chappell, S. L., \& Sexton, G. A. (1986). Advanced concepts flight simulator facility. Applied Ergonomics, 17(4), 252-256.

Federal Aviation Administration (1988). Airman's information manual. U.S. Department of Transportation, Washington, D.C. June 30, 1988, p.C6.S1.10, Table 6-2.

Griffin, M. J. (1990). Handbook of human vibration. San Diego: Academic Press.

Hampton, A. G., \& Dominessy, M. E. (1989). Evaluation of touch and bezel keys as operator input methods in a helicopier environment. Army Human Engineering Laboratory, Aberdeen Proving Ground, MD.

James, D. G. (1987). Man-vehicle research systems facility. National Aeronautics and Space Administration, Ames Research Center, Moffett Field, CA.

Karat, J., McDonald,J., \& Anderson, M. (1986). A comparison of selection techniques: touch panel, mouse, keyboard. International Journal of Man-Machine Studies, 1, 7392.

Lewis, C. H., \& Griffin, M. J. (1976). The effects of vibration on manual control performance. Ergonomics, 19, 203-216. 
Touchscreens and Turbulence

Lewis, C. H., \& Griffin, M. J. (1978). A review of the effects of vibration on visual acuity and continuous manual control, part Iî: Continuous manual control. Journal of Sound and Vibration, 56(3), 415-457.

McLeod, R. W., \& Griffin, M. J. (1986). A design guide for visual and manual tasks in vibration environments, Part II: Manual tasks (ISVR Technical Report No. 134). Southampton S09 5NH, England: University of Southampton, Human Factors Research Unit Institute of Sound and Vibration Research.

Morrocco, J. D. (1991). McDonnell F/A-18E/F offers increased range, endurance. Aviation Week \& Space Technology, March 25, 25-26.

Parks, D. L. (1962). Defining human reaction to whole-body vibration. Human Factors, 4, 305-314.

Potter, R., Berman, M., \& Shneiderman, B. (1989). An experimental evaluation of three touch screen strategies within a hyperties database. International Journal of HumanComputer Interaction 1, 1, 41-52.

Potter, R., Weldon, L., Shneiderman, B. (1988). Improving the accuracy of touch screens: An experimental evaluation of three touch strategies. Proceedings of the ACM Computer Science Conference ;90, ACM, New York, 1-7.

Ruffel Smith, H. P. (1979). A simulator study of the interaction of pilot workload with errors, vigilance, and decision ( NASA TM No. 78482). Washington, DC: National Aeronautics and Space Administration.

Sears, A., Plaisant, C., \& Shneiderman, B. (1991). A New Era for Touchscreen Applications: High Precision, Dragging Icons, and Refined Feedback. To appear in: R. Hartson \& D. Hix (Eds.), Advances in Human-Computer Interaction, Vol 3. 
Touchscreens and Turbulence

Sears, A., \& Shneiderman, B. (1991). High-precision touchscreens: Design strategies and comparisons with a mouse. International Journal of Man-Machine Studies, 34 (4).

Whitfield, D., Ball, R. G., \& Bird, J. M. (1983). Some comparisons of on-display and off-display touch input devices for interaction with computer generated displays.

Ergonomics, 26(11), 1033-1053. 
Touchscreens and Turbulence

\section{Appendix A. Signed Approval Forms}


To: Kristin Bauersfeld, Psychology 4141 Stevenson Blvd. \#355 Fremont, CA 94538

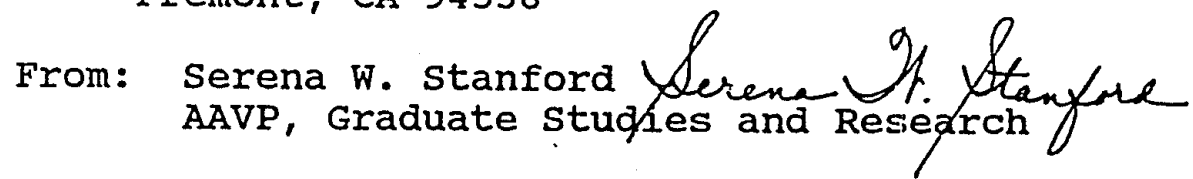

Date: March 18, 1992

The Human Subjects-Institutional Review Board has approved your request to use human subjects in the study entitled:

"Effects of Turbulence and Activation Method on Touchscreen Performance in Aviation Requirements"

This approval is contingent upon the subjects participating in your research project being appropriately protected from risk. This includes the protection of the anonymity of the subjects' identity when they participate in your research project, and with regard to any and all data that may be collected from the subjects. The Board's approval includes continued monitoring of your research by the Board to assure that the subjects are being adequately and properly protected from such risks. If at any time a subject becomes injured or complains of injury, you must notify Dr. Serena stanford immediately. Injury includes but is not limited to bodily harm, psychological trauma and release of potentially damaging personal information.

Please also be advised that each subject needs to be fully informed and aware that their participation in your research project is voluntary, and that he or she may withdraw from the project at any time. Further, a subject's participation, refusal to participate or withdrawal will not affect any services the subject is receiving or will receive at the institution in which the research is being conducted.

If you have questions, please contact me at 408-924-2480.

CC: Kevin Jordan, Ph.D. 
May 27, 1992

TO: Kristin Bauersfeld, MA candidate

FROM: Kevin Jordan, MA Coordinator

RE: Design and analysis review

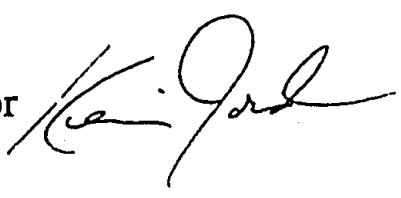

Drs. Fox and Wise have read your thesis proposal for the Design and Analysis

Committee. Their comments are enclosed. Based on their comments, the thesis proposal is approved. As you can see, however, both reviewers have some concerns which must be addressed in the final version of the thesis.

Based on this committee's approval, the collection of data for your thesis is approved contingent on documentation of compliance with university policy regarding the use of human subjects in research. University policy requires approval of your project by the Human Subjects Institutional Review Board. Please provide me with a file copy documenting such approval as soon as you receive it. After that copy is part of your file, you may begin collecting data.

Congratulations on your progress to date! We look forward to the continuation of your fine performance in the program.

cc:

Cooper

Degani (NASA-ARC; forward to Jordan)

Fox

Jordan

Wise

file 